\title{
Optimising the performance of the CMS Electromagnetic Calorimeter to measure Higgs properties during Phase I and Phase II of the LHC
}

\author{
Davide Valsecchi* \\ Università degli Studi di Milano-Bicocca, CERN \\ E-mail: davide.valsecchi@cern.ch
}

The CMS Electromagnetic Calorimeter (ECAL), is a high granularity lead tungstate crystal calorimeter operating at the CERN LHC. The original design placed a premium on excellent energy resolution. Excellent energy resolution and efficient identification for photons are essential to reconstruct the Higgs boson in the $H \rightarrow \gamma \gamma$ decay channel, for measurements of the self-coupling of Higgs bosons and other related parameters.

The ECAL performance has been crucial in the discovery and subsequent characterisation of the Higgs boson. The original ECAL design considerations, and the actual experimental energy reconstruction and calibration precision will be reviewed.

The improvements to the energy reconstruction and energy calibration algorithms for LHC Run II are described. These are required to maintain the stability of the ECAL energy scale and resolution for the higher LHC luminosities that have been experienced compared to Run I. The precision measurement of the Higgs decay modes is central to the HL-LHC physics program. In addition, the search for di-Higgs production is important to understand the details of the vacuum potential. The crystals in the barrel region will be retained for HL-LHC. The decrease of operating temperature and upgrades to the readout electronics that are needed to maintain the required performance of the barrel region from 2026 onwards will be described.

These upgrades will ensure that radiation-induced noise increases will not dominate the energy resolution for photons from Higgs boson decays, and will preserve the ability of CMS to trigger efficiently on these signals. They will also permit precision time measurements (30 ps rms error on the arrival time of photons from Higgs boson decays) which will improve the determination of the location of the production vertex for di-photon events. Time measurement performance of the new readout electronics has been characterized in beam tests.

The predicted electron and photon energy resolution and identification efficiencies expected for HL-LHC will be described, and the performance relevant to a number of key Higgs decay channels will be presented.

European Physical Society Conference on High Energy Physics - EPS-HEP2019 -

10-17 July, 2019

Ghent, Belgium

* On behalf of the CMS Collaboration 


\section{The CMS Electromagnetic Calorimeter (ECAL)}

The CMS electromagnetic calorimeter [1] is a homogeneous calorimeter made of 75848 lead tungstate $\left(\mathrm{PbWO}_{4}\right)$ scintillating crystal, located inside the CMS superconducting solenoid magnet. It is made of a barrel part (EB) covering the region of pseudorapidity $|\eta|<1.48$ with 61200 crystals and two endcaps (EE), which extend the coverage up to $|\eta|<3.0$ with 7324 crystals each. Scintillation light is detected with Avalanche Photodiodes (APD) in the barrel and Vacuum Phototriodes (VPT) in the endcaps. The calorimeter is crucial for the identification and reconstruction of photons and electrons, and for the measurement of jets and missing transverse momentum. Electrons and photons are typically reconstructed up to $|\eta|<2.5$, the region covered by the tracker, while jets are reconstructed up to $|\eta|<3.0$.

\section{Performances during Run II}

The ECAL high resolution and efficient identification of photons during Run I has been fundamental for the discovery of Higgs boson in the $H \rightarrow \gamma \gamma$ decay channel. During Run II (2015-2018), the LHC delivered proton-proton collisions at an increased centre of mass energy of $13 \mathrm{TeV}$, with colliding proton bunches every $25 \mathrm{~ns}$. The beam intensities were significantly higher than in Run I, reaching $1.7 \times 10^{34} \mathrm{~cm}^{-2} \mathrm{~s}^{-1}$, with up to 60 concurrent interactions per bunch crossing (termed pileup). By the end of Run II, the LHC delivered an integrated luminosity of almost $160 \mathrm{fb}^{-1}$, and the CMS ECAL operated with more than $99 \%$ of its channels active, and achieved higher than 99.5\% operational efficiency (by luminosity).

These new challenging operation conditions made necessary a retuning of the ECAL readout and trigger threshold and reconstruction algorithms, to maintain the best possible performance in these more challenging conditions. The energy response of the detector must be precisely calibrated and monitored to achieve and maintain the excellent performance obtained in Run I in terms of energy scale and resolution.

The main concern about high instantaneous luminosity is that ECAL energy response is influenced by radiation. The lead tungstate crystals are intrinsic scintillators: the radiation doesn't affect the main scintillation mechanism, instead it creates crystals defects which reduce the crystal transparency decreasing the light output. Defects caused by electromagnetic radiation anneals spontaneously with time, whereas hadronic damage creates permanent loss of crystal transparency. Therefore, during LHC operation the transparency of each ECAL crystal is monitored and corrected using a dedicated light injection monitoring system [2]. As shown in Figure 1a, at the end of Run II the response change observed in the ECAL channels is up to $13 \%$ in the barrel and it reaches up to $62 \%$ at $|\eta| \sim 2.5$, the limit of the tracker acceptance. The recovery of the crystal response during the periods without collisions is visible. These measurements, performed every 40 minutes, are used to correct the physics data.

The readout of an ECAL channel is a set of 10 amplitude samples, enough to cover the amplified signal pulse. The time spacing between two consecutive samples is $25 \mathrm{~ns}$, the same time spacing between two colliding bunches in the LHC. Therefore, there can be more pulses overlapping to the signal in a single readout. An amplitude reconstruction algorithm, termed "MultiFit", has been developed and used during Run II to model the signal pulse shape as a sum of one in-time 
pulse and a series of out-of-time (OOT) pulses. A pulse fit is performed to extract the amplitude of the in-time and OOT pulses, given the crystal pulse shape and the noise correlation matrices which are periodically measured in dedicated runs [3]. This technique makes negligible the contribution of out-of-time energy to the energy reconstruction. The energy resolution improvement is substantial especially for low energy photons and electrons, given the larger contribution of deposits from pileup to the total energy.

A dedicated calibration of each detector channel is performed with physics events exploiting electrons from $\mathrm{W}$ and $\mathrm{Z}$ boson decays, photons from $\pi_{0}$ and $\eta$ decays, and from the azimuthally symmetric energy distribution of minimum bias events. Moreover, the variation along time of several detector parameters (as electronic pedestals, noise, reference response to the laser light) has been carefully measured. Refined corrections have been implemented for the legacy reconstruction of all the Run II data resulting in excellent energy resolution along all detector regions (up to $\sim 2 \%$ in the central region of EB) as shown in Figure 1b for electrons from $\mathrm{Z}$ decays [4].

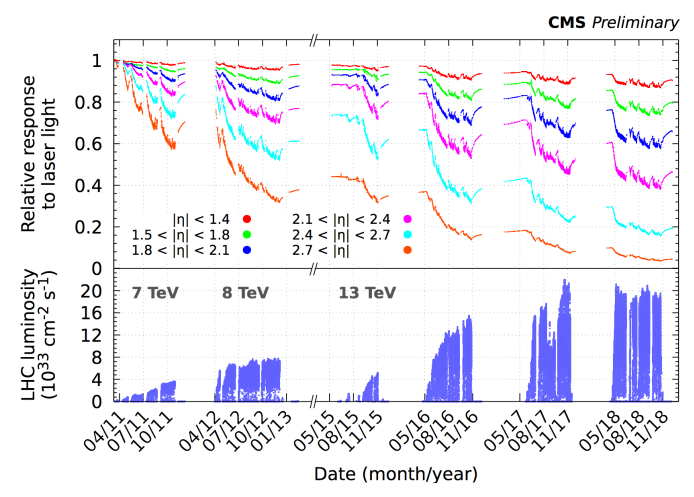

(a) Relative response to laser light injected in the ECAL crystals, measured by the ECAL laser monitoring system, averaged over all crystals in bins of pseudorapidity $(\eta)$.

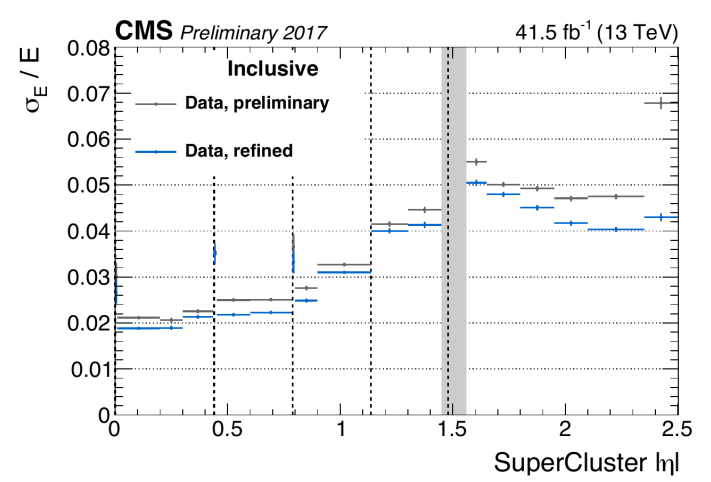

(b) Relative electron (ECAL) energy resolution unfolded in bins of pseudo-rapidity $\eta$ for EB and EE. All electrons from $\mathrm{Z} \rightarrow$ ee decays are used (inclusive).

\section{ECAL Phase II Upgrade}

The main objective of the High Luminosity LHC (HL-LHC) [5] upgrade of the LHC accelerator is to deliver a much larger dataset for physics to the LHC experiments, for new physics searches, Higgs boson coupling measurements and precision tests of the standard model. The baseline operating parameters for HL-LHC include a levelled luminosity of $5 \times 10^{34} \mathrm{~cm}^{-2} \mathrm{~s}^{-1}$, a peak pileup of 140 , a delivered luminosity of $250 \mathrm{fb}^{-1}$ per year, for a total integrated luminosity of $3000 \mathrm{fb}^{-1}$ by 2038.

The existing CMS calorimeters were designed to sustain up to $500 \mathrm{fb}^{-1}$ and need to be upgraded for HL-LHC. The EB photodetectors and lead tungstate crystals will be retained, whereas the front-end and off-detector electronics will be re-designed and replaced. The ECAL endcaps will suffer significant radiation damage, necessitating a complete replacement at the end of Run III [6]. The principal requirement for the EB upgrade is to maintain the Run 1 physics performance for photons and electron at higher luminosity and pileup of the HL-LHC. In order to be ready for it, 
the EB electronics needs to fit the Level-1 trigger requirements on latency and rate, provide more precise timing resolution, and mitigate the increasing noise from the photodetectors.

The primary technical motivation for the EB upgrade is the Level-1 trigger requirements. The current ("legacy") system provides trigger primitives of $5 \times 5$ crystals with a maximum latency of $4 \mu s$ and a rate of $100 \mathrm{kHz}$. The upgrade will instead provide single crystal information to the Level-1 calorimeter trigger with a maximum latency of $12.5 \mu \mathrm{s}$ and a rate up to $750 \mathrm{kHz}$, in order to precisely match electromagnetic showers to tracks and to provide better isolation compared to the current system. This will significantly reduce backgrounds and enable the calorimeter trigger threshold to remain at the levels required for precision study of the Higgs boson [6].

The Very Front End (VFE) card, which provides pulse amplification, shaping and digitization functions, must be replaced to provide better timing resolution and noise filtering. Shortening the pulse shaping time and increasing the sampling rate from $40 \mathrm{MHz}$ to $160 \mathrm{MHz}$ will more optimally filter the increased APD noise and will improve considerably the timing resolution. This will allow anomalous signals in the APDs (termed "spikes" [7]) to be almost completely suppressed in the Level-1 trigger by their different time development compared to the scintillation light. The legacy system only provides minimal time discrimination and without additional timing and isolation information the Level-1 trigger would be saturated by spikes during Phase II.

The increasing level of dark current in the APDs caused by radiation damage will be also kept under control decreasing the operating temperature from $18{ }^{\circ} \mathrm{C}$ to $9{ }^{\circ} \mathrm{C}$ : this will reduce the dark current to $50 \%$ of its value [6].

The baseline solution for VFE implementation is a Trans-Impedance Amplifier (TIA) that outputs a voltage image of the photocurrent generated by the APD. Two output gains are used to cover the full dynamic range of the signal up to $2 \mathrm{TeV}$. The best performance for timing resolution and spike rejection is achieved if the signal is sampled at $160 \mathrm{MHz}$ [6].

The amplifier ASIC is followed by a data compression and transmission ASIC, called LiTEDTU (Lisbon-Torino ECAL Data Transmission Unit). The LiTE-DTU receives the two analog signals from the preamplifier outputs (two gains) and converts them to a digital representation of the pulse with a commercial ADC. For each readout window, either the high or the low gain is selected and sent to the off-detector electronics on the lpGBT [8] e-link protocol. More then 99\% of the events can be codified in 6-7 bits and a lossless data compression scheme will be applied to reduce the data output bandwidth.

In the current system Level-1 trigger primitives are formed on-detector and transmitted offdetector while the full-granularity data waits in limited buffers on the front-end electronics. In the upgraded system all the digitized samples will be transmitted off-detector for trigger primitive generation and data processing by commercially available powerful FPGAs. A processor board will be designed with these FPGAs and high speed optical links following the Advanced Telecommunications Computing Architecture (ATCA) standard [9]. The board will analyse incoming data and transmit a pre-processed set of trigger primitives to the next layer of the Level-1 trigger system. The algorithms will include the rejection of spikes and basic clustering of localized energy.

\section{Expected performance during HL-LHC}

The MultiFit amplitude reconstruction algorithm used during LHC Run II is also expected to 
be used for HL-LHC since it is effective in suppressing OOT PU from collisions with $<$ PU $>=$ 200 to the level of the intrinsic electronic noise, $\sigma_{\text {noise }}$, for values of $\sigma_{\text {noise }}>10 \mathrm{MeV}$ [6]. The energy resolution can be estimated as the sum of three independent contributions described by noise, stochastic, and constant terms. Figure 2a shows the estimated energy resolution after 1000 $\mathrm{fb}^{-1}$ at HL-LHC including the simulation of light output loss of the crystals, APD dark current increase, upgraded electronics and lowered operating temperature. The upgrade of the front-end electronics will keep the noise term small and all the intercalibration procedures developed during Run II will be updated and improved to minimize the constant term.

The algorithm used in Run II for ECAL timing reconstruction will be adapted to the new TIA pulse shape and increased sampling rate. The method uses ratios of subsequent samples [10] to measure the timing of the signal pulse. A timing resolution of better then $30 \mathrm{ps}$ has been obtained at a test beam using prototype TIA electronics as shown in Fig. $2 b$.

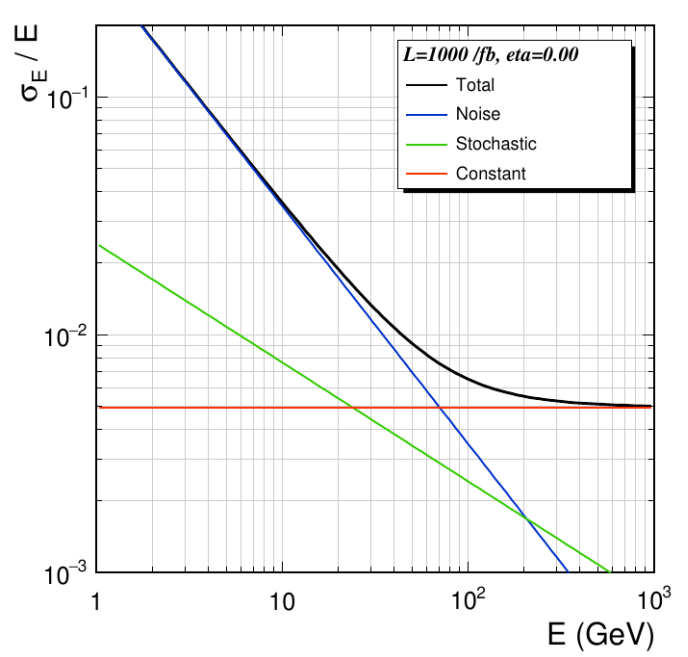

(a) Energy resolution as a function of incident particle energy after detector ageing up to an integrated luminosity of $1000 \mathrm{fb}^{-1}$ at $|\eta|=0$.

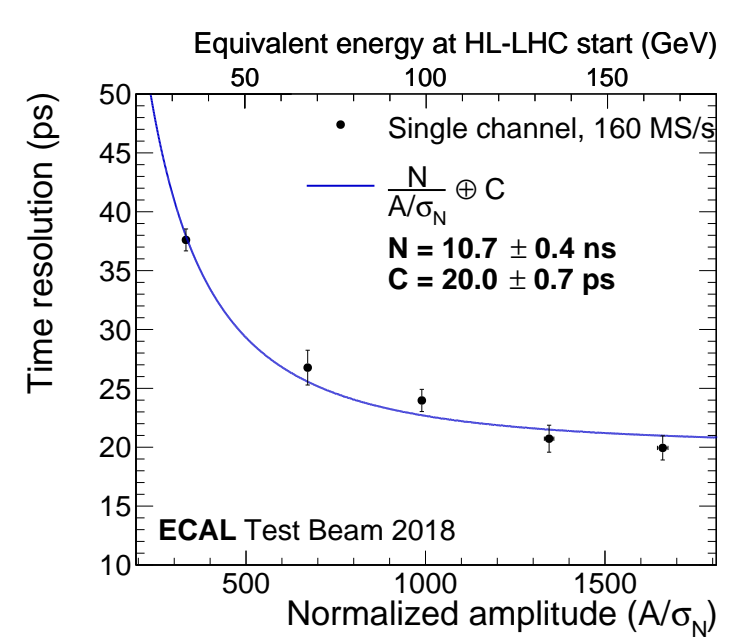

(b) Timing resolution function of the noise normalized amplitude obtained at test beam using prototype TIA at $18^{\circ} \mathrm{C}$.

Electron and photon identification strategies will be similar to Run II ones during HL-LHC and multivariate techniques will be implemented combining calorimeter and track information. Simulations in HL-LHC conditions show that even with pileup of 200, physics performance comparable to Run II can be achieved [6].

The degradation in photon and electron energy resolution is mainly due to pileup local fluctuations rather that detector ageing, event after $4500 \mathrm{fb}^{-1}$ of integrated luminosity [6]. The reconstruction is currently not optimized for a pileup of 200 and further developments are ongoing to maintain the optimal performance of Run II.

The resolution of the $\mathrm{H} \rightarrow \gamma \gamma$ invariant mass depends on the resolution of the photon energy measured in the calorimeter and the resolution on the opening angle between the two photons. If the interaction point is known to better than $10 \mathrm{~mm}$, the contribution from resolution on the opening angle between the photons is negligible, compared to the energy resolution of the calorimeter. The efficiency to identify the correct vertex within $1 \mathrm{~cm}$ goes down from $40 \%$ to $30 \%$ if the PU goes from 140 to 200. The predictions show that precise measurement of the time of flight of photons 
in $\mathrm{EB}$, with a resolution better than $30 \mathrm{ps,} \mathrm{will} \mathrm{improve} \mathrm{the} \mathrm{di-photon} \mathrm{mass} \mathrm{resolution} \mathrm{by} \mathrm{means} \mathrm{of}$ precise measurement of the vertex position along the beam direction determined by triangulation.

The reviewed ECAL Barrel upgrade will make possible to perform challenging CMS analyses at HL-LHC thanks to expected excellent energy resolution, high efficiency for the detection of electrons and photons, fast response, and high granularity to mitigate the effect the pileup.

\section{References}

[1] CMS collaboration, The CMS experiment at the CERN LHC, Journal of Instrumentation 3 (2008) S08004.

[2] M. Anfreville et al., Laser monitoring system for the CMS lead tungstate crystal calorimeter, Nucl. Instrum. Meth. A594 (2008) 292.

[3] CMS collaboration, ECAL hit timing with Run1 and Run2 energy reconstruction in early 25ns data at $13 \mathrm{TeV}, C M S-D P-2015-040(2015)$.

[4] CMS collaboration, CMS ECAL performance with 2017 data, CMS-DP-2019-029 (2019) .

[5] Apollinari G. and Béjar Alonso I. et al., High-Luminosity Large Hadron Collider (HL-LHC): Technical Design Report V. 0.1, CERN Yellow Reports: Monographs. CERN, Geneva, 2017, 10.23731/CYRM-2017-004.

[6] CMS collaboration, The Phase-2 Upgrade of the CMS Barrel Calorimeters, Tech. Rep. CERN-LHCC-2017-011. CMS-TDR-015, CERN, Geneva, Sep, 2017.

[7] D. A. Petyt, Mitigation of anomalous APD signals in the CMS electromagnetic calorimeter, Journal of Physics: Conference Series 404 (2012) 012043.

[8] Moreira, P and Ballabriga, et al., The GBT Project, http://cds.cern.ch/record/1235836 (2009) .

[9] Advanced TCA base specification: advanced TCA. PICMG, Wakefield, MA, 2008.

[10] CMS collaboration, Time Reconstruction and Performance of the CMS Electromagnetic Calorimeter, JINST 5 (2010) T03011 [0911. 404 4]. 